\title{
Do surgimento à extinção: a trajetória de um serviço ambulatorial de genética médica no Brasil
}

\author{
From emergence to extinction: the trajectory of an outpatient medical genetics service in \\ Brazil
}

\author{
Tatiane de Abreu ${ }^{1}$ \\ Orcid: http://orcid.org/0000-0003-2534-4064 \\ Heloisa Pastana Marsiglio ${ }^{3}$ \\ Orcid: http://orcid.org/0000-0001-5566-5294 \\ Natália Santander Ortensi ${ }^{5}$ \\ Orcid: http://orcid.org/0000-0002-9385-8967
}

Érica Letícia Angelo Liberato ${ }^{2}$

Thamires Rosa dos Santos ${ }^{4}$

Orcid: http://orcid.org/0000-0002-6177-2632

\author{
Calógeras A. de Albergaria Barbosa ${ }^{6}$ \\ Orcid: http://orcid.org/0000-0002-7759-4033
}

\author{
Débora Gusmão Melo ${ }^{7}$ \\ Orcid: http://orcid.org/0000-0001-7005-3544
}

\begin{abstract}
Resumo
Introdução: $\mathrm{O}$ número de profissionais de saúde envolvidos na assistência especializada em genética é considerado insuficiente no Brasil, e supõe-se que a maior parte dos pacientes e famílias não recebe cuidado adequado. Além disso, a disponibilidade de recursos humanos e material para atendimento em genética é muito desigual nas várias regiões do país. Objetivos: Descrever a trajetória de um serviço ambulatorial de genética médica no Brasil e ressaltar a importância de organizar a rede de atenção em genética médica no país. Metodologia: Estudo quantitativo, transversal e retrospectivo, no qual foram analisados os mapas de atendimento de todos os 792 pacientes avaliados no Ambulatório de Genética Médica de São Carlos, São Paulo, entre junho de 2006 e dezembro de 2018. Resultados: Houve grande diversidade de doenças, genéticas, que acometeram pessoas de diferentes faixas etárias. Um diagnóstico genético foi definido em 290 pacientes (36,6\%) e afastado em 153 pacientes (19,3\%); 23 pacientes $(2,9 \%)$ receberam aconselhamento genético para futura descendência e em 326 pacientes $(41,2 \%)$ um diagnóstico genético não foi definido nem afastado. A frequência de casos não resolvidos explicita dificuldades inerentes ao diagnóstico clínico genético e barreiras para acesso aos testes genéticos moleculares. Conclusão: Experiências como a do Ambulatório de Genética Médica de São Carlos mostram os obstáculos de se prestar um serviço de saúde adequado à população, reforçando a necessidade de organizar a rede de atenção em genética no Sistema Único de Saúde, com base na Política Nacional de Atenção Integral às Pessoas com Doenças Raras.
\end{abstract}

Palavras-chave: genética médica; aconselhamento genético; doenças raras; encaminhamento e consulta; pesquisa sobre serviços de saúde; Sistema Único de Saúde.

\footnotetext{
${ }^{1}$ Universidade Federal de São Carlos (UFSCar), São Carlos, SP, Brasil. E-mail: tatianeabreu10@hotmail.com

${ }^{2}$ Universidade Federal de São Carlos (UFSCar), São Carlos, SP, Brasil. E-mail: leticialiberato@gmail.com

${ }^{3}$ Universidade Federal de São Carlos (UFSCar), São Carlos, SP, Brasil. E-mail: helopastana@hotmail.com

${ }^{4}$ Universidade Federal de São Carlos (UFSCar), São Carlos, SP, Brasil. E-mail: thami rosa.29@hotmail.com

${ }^{5}$ Universidade Federal de São Carlos (UFSCar), São Carlos, SP, Brasil. E-mail: ortensinatalia@gmail.com

${ }^{6}$ Universidade Federal de São Carlos (UFSCar), São Carlos, SP, Brasil. E-mail: cabaciam@gmail.com

${ }^{7}$ Universidade Federal de São Carlos (UFSCar), São Carlos, SP, Brasil. E-mail: dgmelo@ufscar.br
} 


\begin{abstract}
Introduction: The number of health professionals involved in specialized care in genetics is considered insufficient in Brazil, and it is assumed that most patients and families do not receive adequate care. Also, the availability of human and material resources for care in genetics is very uneven in various regions of the country. Objectives: To describe the trajectory of an outpatient medical genetics service in Brazil and to highlight the importance of organizing the medical genetic care network in this country. Methodology: Quantitative, cross-sectional, and retrospective study, in which the care maps of all 792 patients evaluated at Medical Genetics Outpatient Clinic in São Carlos, São Paulo, from June 2006 to December 2018 were analyzed. Results: There was a great diversity of genetic diseases, which affect people in different age groups. A genetic diagnosis was defined in 290 patients $(36.6 \%)$ and ruled out in 153 patients $(19.3 \%)$; 23 patients $(2.9 \%)$ received genetic counseling for future offspring and in 326 patients $(41.2 \%)$ a genetic diagnosis was neither defined nor ruled out. The frequency of unresolved cases explains difficulties inherent to clinic genetic diagnosis and barriers to access molecular genetic tests. Conclusion: Experiences such as Medical Genetics Outpatient Clinic in São Carlos show the obstacles to providing adequate health services to the population in genetics, reinforcing the need to organize the genetic care network in the Brazilian Unified Health System, based on the National Policy for Comprehensive Care for People with Rare Diseases.
\end{abstract}

Keywords: medical genetics; genetic counseling; rare diseases; referral and consultation; health services research; Brazilian Unified National Health System.

\section{Introdução}

As doenças genéticas são numerosas e diversas. Estima-se que existam entre 6.000 a 7.000 doenças genéticas diferentes, que, conjuntamente, atingem cerca de 31,5 a 73 por 1.000 indivíduos da população geral $^{1}$. Elas podem ser congênitas ou se desenvolver ao longo da vida, ocorrendo em todas as faixas etárias. Muitas são hereditárias, acometendo vários indivíduos de uma mesma família, outras surgem por mutação nova, sem que haja antecedente familiar. Geralmente são crônicas, graves, progressivas e, além de todo impacto pessoal e familiar, acarretam prejuízo socioeconômico para o país, pois elevam os gastos com saúde e diminuem a produtividade ${ }^{2}$.

No Brasil, o número de profissionais de saúde envolvidos na assistência especializada em genética é considerado insuficiente e supõe-se que a maior parte dos pacientes e famílias não recebe cuidado adequado $^{3}$. Em 2020, havia 332 médicos com título de especialista em genética, registrados no Conselho Federal de Medicina $^{4}$. Além de serem poucos, os especialistas, assim como os serviços de genética, estão concentrados de maneira bastante desigual, com predomínio nas regiões Sudeste e $\mathrm{Sul}^{4-7}$. A escassez de recursos humanos e serviços especializados é, indubitavelmente, um obstáculo para inclusão da assistência em genética médica no SUS ${ }^{3,6}$.

Uma primeira tentativa de incluir a genética no SUS aconteceu em 2009, com a publicação da Política Nacional de Atenção Integral em Genética Clínica ${ }^{8}$ que, no entanto, não foi efetivamente implantada. Em 2014, foi instituída a Política Nacional de Atenção Integral às Pessoas com Doenças Raras ${ }^{9}$ e, como cerca de $70 \%$ das doenças raras têm etiologia genética ${ }^{2}$, surgiu uma nova oportunidade de se organizar a rede de atenção em genética médica no SUS ${ }^{3,10}$. A Política Nacional de Atenção Integral às Pessoas com Doenças Raras estabelece as ações que devem ser desenvolvidas pela atenção básica e aquelas que devem ser desenvolvidas 
especificamente pela atenção especializada. Para isso, esta Política reconhece e habilita os Serviços de Atenção Especializada em Doenças Raras e os Serviços de Referência em Doenças Raras 9.

Levantamento realizado no site da Internet da Sociedade Brasileira de Genética Médica e Genômica ${ }^{11}$ permitiu identificar 108 serviços públicos de genética médica, incluindo serviços hospitalares de alta complexidade e ambulatórios de doenças específicas, distribuídos de forma desigual: 56 serviços no Sudeste, 23 no Nordeste, 15 no Sul, 8 no Norte e 5 no Centro-Oeste. A maioria destes serviços é integrada a hospitais e/ou instituições universitárias públicas.

A despeito da demanda reprimida de pessoas que necessitam de atendimento especializado na área, até dezembro de 2020 o Ministério da Saúde havia habilitado apenas um "Serviço de Atenção Especializada em Doenças Raras" e 16 "Serviços de Referência em Doenças Raras", em consonância com a Política Nacional de Atenção Integral às Pessoas com Doenças Raras, em todo território nacional ${ }^{11}$. Especialmente nos serviços que não estão habilitados, há muita dificuldade para acesso aos testes genéticos, que geralmente são oferecidos em contexto de pesquisa e realizados apenas durante determinado período, não se mantendo oferta contínua ao longo do tempo ${ }^{6,7}$.

No município de São Carlos, interior do Estado de São Paulo, em 2006, foi organizado um serviço ambulatorial de genética médica, como um projeto de extensão universitária, fazendo parte da rede saúde-escola ${ }^{12}$. Este serviço funcionou regularmente até dezembro de 2018, quando os atendimentos foram descontinuados. O objetivo do presente estudo foi descrever a trajetória do Ambulatório de Genética Médica de São Carlos, São Paulo, e realizar uma análise retrospectiva do trabalho conduzido durante o período de funcionamento, caracterizando a população atendida, com o intuito de ressaltar e discutir a importância de organizar a rede de atenção em genética no país.

\section{Metodologia}

Este é um estudo de abordagem quantitativa, de corte transversal e retrospectivo, desenvolvido após aprovação pelo Comitê de Ética em Pesquisa com Seres Humanos da Universidade Federal de São Carlos (UFSCar) (CAAE 66080517.2.0000.5504). A cidade de São Carlos está localizada no centro geográfico do Estado de São Paulo, Brasil; possui população estimada de cerca de 250.000 habitantes e, em 2010, apresentou índice de desenvolvimento humano de 0,805 , sendo considerado o $28^{\circ}$ município mais desenvolvido do país ${ }^{13}$.

O Ambulatório de Genética Médica de São Carlos funcionou entre junho de 2006 e dezembro de 2018, no espaço físico do Centro Municipal de Especialidades em Saúde (CEME), com atendimentos semanais, realizados conjuntamente por dois médicos geneticistas, ambos professores universitários, frequentemente acompanhados por estudantes de medicina. Oficialmente, eram disponibilizadas duas vagas de atendimento para pacientes novos e três vagas de atendimento para retorno de pacientes que já estavam em seguimento ambulatorial, embora, eventualmente, outros arranjos na composição destas vagas tenham sido feitos ao longo dos anos.

O Ambulatório era o único serviço de genética médica público no município, atendendo também a demanda de outras oito cidades da microrregião ${ }^{12}$ e totalizando uma área de abrangência de cerca de 530.000 pessoas $^{13}$.

$\mathrm{O}$ único teste genético oferecido rotineiramente no Ambulatório durante todo período de funcionamento foi $\mathrm{o}$ cariótipo convencional, realizado por meio da contratação de serviços laboratoriais terceirizados pela Secretaria Municipal de Saúde, uma vez que este exame 
complementar é um procedimento com finalidade diagnóstica que consta na tabela SUS. O teste era feito a partir de cultura de linfócitos de sangue periférico com coloração por bandeamento $\mathrm{G}$ e resolução entre 400-550 bandas. $\mathrm{O}$ estudo citogenético era realizado nos pacientes atendidos no Ambulatório mediante as seguintes indicações clínicas: suspeita de síndromes cromossômicas clássicas; defeitos congênitos múltiplos, com ou sem presença de dismorfias faciais; deficiência intelectual ou atraso global de desenvolvimento de etiologia não definida, com ou sem dismorfias faciais; baixa estatura patológica em meninas; ambiguidade genital; infertilidade; amenorreia primária e abortamento de repetição.

Para análise retrospectiva dos atendimentos, a coleta de dados foi realizada por meio de análise dos "Mapas Diários de Atendimento Médico" dos pacientes atendidos no Ambulatório entre junho de 2006 e dezembro de 2018. Não houve critério de exclusão, tendo sido incluídos na revisão todos os "Mapas" dos pacientes atendidos no período citado. Os "Mapas Diários de Atendimento Médico" são fichas preenchidas diariamente, após os atendimentos clínicos ambulatoriais, com informações básicas sobre essas consultas. A partir dos "Mapas" revisados foram extraídas informações sobre: ano do atendimento; idade e sexo do paciente; motivo do encaminhamento; e diagnóstico genético, quando definido.

Os dados coletados foram categorizados e analisados por meio de estatística descritiva. Os resultados foram discutidos e examinados com auxílio da literatura e da experiência clínica dos autores, através de uma abordagem interpretativa e compreensiva.

\section{Resultados}

No período estudado foram atendidos 792 pacientes diferentes, sendo que 48 pacientes $(6 \%)$ eram aparentados. Com relação ao sexo, 397 (50,1\%) eram do sexo feminino, $385(48,6 \%)$ do sexo masculino e em 10 situações $(1,3 \%)$ o sexo do paciente não foi determinado por incompletude de informação. A idade variou entre 5 dias de vida e 79 anos (Figura 1), com predominância de população jovem: $71,5 \%$ dos pacientes tinham até 20 anos. 
Figura 1: Distribuição dos 792 pacientes atendidos no Ambulatório de Genética Médica de São Carlos, SP, entre 2006 e 2018, por faixa etária.

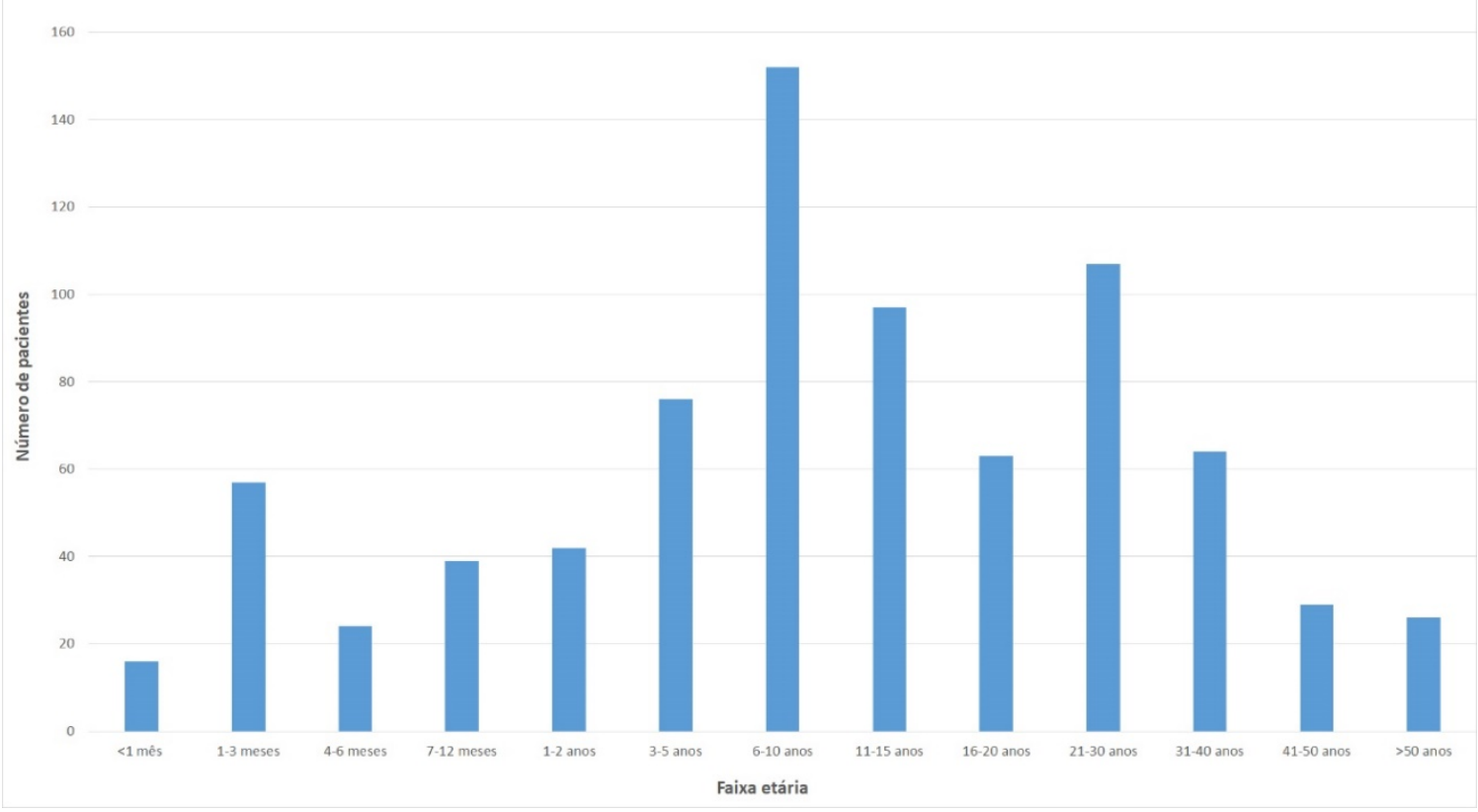

A distribuição dos atendimentos ao longo dos anos (Figura 2) revelou uma interrupção de casos novos durante o ano de
2011 e a diminuição deste número no ano de 2018.

Figura 2: Distribuição do número de novos pacientes atendidos no Ambulatório de Genética Médica de São Carlos, SP, entre 2006 e 2018, ao longo dos anos.

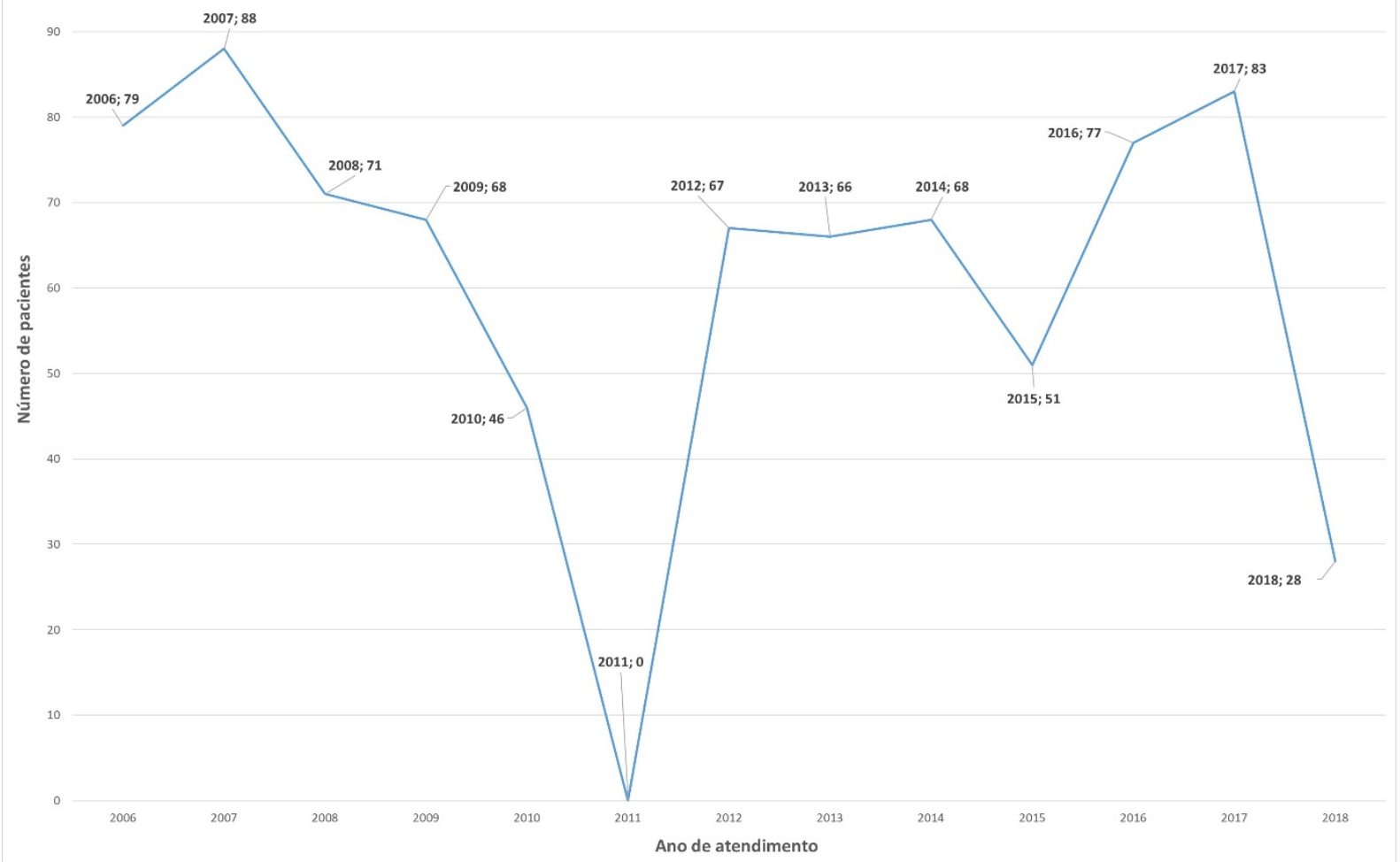


Os motivos de encaminhamento dos diferentes pacientes foram categorizados e são apresentados na Tabela 1. As Tabelas 2, 3 e 4 apresentam os diagnósticos genéticos nas 290 situações $(36,6 \%)$, nas quais este foi definido.

Tabela 1: Motivos de encaminhamento inicial para o Ambulatório de Genética Médica de São Carlos, SP, entre 2006 e $2018(\mathrm{~N}=792)$.

\begin{tabular}{|c|c|c|}
\hline MOTIVO DE ENCAMINHAMENTO & $\mathbf{N}$ & $\%$ \\
\hline Deficiência intelectual isolada ou com pequenas dismorfias faciais & 207 & 26,1 \\
\hline Leve & 55 & 6,9 \\
\hline Moderada & 93 & 11,7 \\
\hline Grave à profunda & 59 & 7,4 \\
\hline Malformações múltiplas, incluindo ou não deficiência intelectual & 144 & 18,2 \\
\hline Abortamento de repetição & 46 & 5,8 \\
\hline Malformação congênita isolada & 43 & 5,4 \\
\hline Baixa estatura & 43 & 5,4 \\
\hline Suspeita de genodermatose/hamartomatose & 40 & 5,0 \\
\hline Atraso global de desenvolvimento isolado em crianças menores de 5 anos & 40 & 5,0 \\
\hline Suspeita clínica de síndrome de Down & 37 & 4,7 \\
\hline Aconselhamento genético para descendência & 28 & 3,5 \\
\hline História de óbito perinatal de feto com múltiplos defeitos congênitos & 14 & 1,8 \\
\hline Consanguinidade do casal & 6 & 0,7 \\
\hline História familiar positiva de doença genética previamente diagnosticada & 6 & 0,7 \\
\hline Idade materna avançada & 2 & 0,2 \\
\hline Suspeita de doença de tecido conjuntivo e/ou habitus marfanoide & 26 & 3,3 \\
\hline Criança com dismorfias faciais, sem disfunções ou malformações maiores & 25 & 3,1 \\
\hline Suspeita de doença neurogenética & 21 & 2,6 \\
\hline Transtorno de espectro do autismo sem deficiência intelectual & 17 & 2,1 \\
\hline Deficiência visual & 17 & 2,1 \\
\hline Suspeita de síndrome de câncer hereditário & 15 & 1,9 \\
\hline Infertilidade masculina & 13 & 1,6 \\
\hline Surdez ou perda auditiva importante & 10 & 1,3 \\
\hline Osteocondrodisplasia & 7 & 0,9 \\
\hline Distúrbio de diferenciação e/ou determinação sexual & 5 & 0,6 \\
\hline Atraso puberal feminino e/ou amenorreia primária & 4 & 0,5 \\
\hline Atraso puberal masculino & 3 & 0,4 \\
\hline Avaliação de criança resultado de relação consanguínea incestuosa & 1 & 0,1 \\
\hline
\end{tabular}

Tabela 2: Distribuição dos pacientes com alterações cromossômicas, atendidos no Ambulatório de Genética Médica de São Carlos, SP, entre 2006 e 2018, nos quais foi definido um diagnóstico genético (N=77).

\begin{tabular}{|c|c|c|}
\hline DIAGNÓSTICO GENÉTICO & $\mathbf{N}$ & $\%$ \\
\hline \multicolumn{3}{|l|}{ Alterações cromossômicas identificadas pelo cariótipo convencional } \\
\hline Cromossomopatias numéricas & 58 & 20,0 \\
\hline Síndrome de Down & 33 & 11,4 \\
\hline Síndrome de Edwards & 5 & 1,7 \\
\hline Síndrome de Patau & 2 & 0,7 \\
\hline Síndrome de Turner & 6 & 2,1 \\
\hline Síndrome de Klinefelter & 6 & 2,1 \\
\hline Síndrome de duplo Y & 3 & 1,0 \\
\hline Síndrome de triplo $\mathrm{X}$ & 1 & 0,3 \\
\hline Anormalidades de cromossomos não definidos (marcadores cromossômicos) & 2 & 0,7 \\
\hline Polimorfismos cromossômicos & 7 & 2,4 \\
\hline Cromossomopatias estruturais desequilibradas & 6 & 2,1 \\
\hline Cromossomopatias estruturais equilibradas & 6 & 2,1 \\
\hline
\end{tabular}


Tabela 3: Distribuição dos pacientes com doenças genéticas não cromossômicas, atendidos no Ambulatório de Genética Médica de São Carlos, SP, entre 2006 e 2018, nos quais foi definido um diagnóstico genético (N=165).

\begin{tabular}{|c|c|c|}
\hline DIAGNÓSTICO GENÉTICO & $\mathbf{N}$ & $\%$ \\
\hline \multicolumn{3}{|l|}{ Doenças genéticas não cromossômicas } \\
\hline Síndromes multissistêmicas & 58 & 20,0 \\
\hline Síndrome de X-frágil & 8 & 2,7 \\
\hline Síndrome de Crouzon & 7 & 2,4 \\
\hline Síndrome de Prader-Willi & 5 & 1,7 \\
\hline Síndrome de Cornelia de Lange & 4 & 1,4 \\
\hline Síndrome de Silver-Russel & 3 & 1,0 \\
\hline Síndrome de Robinow & 3 & 1,0 \\
\hline Síndrome de Williams & 3 & 1,0 \\
\hline Síndrome de Stickler & 2 & 0,7 \\
\hline Síndrome de microdeleção 22q11.2 & 2 & 0,7 \\
\hline Síndrome de Joubert & 2 & 0,7 \\
\hline Síndrome de Sotos & 2 & 0,7 \\
\hline Síndrome de Goldenhar & 2 & 0,7 \\
\hline Síndrome de Kartagener & 1 & 0,3 \\
\hline Síndrome de Waardenburg & 1 & 0,3 \\
\hline Síndrome de Seckel & 1 & 0,3 \\
\hline Síndrome de Beckwith-Wiedemann & 1 & 0,3 \\
\hline Síndrome de Lowe & 1 & 0,3 \\
\hline Síndrome de Peters Plus & 1 & 0,3 \\
\hline Mucopolissacaridose tipo IV-A & 1 & 0,3 \\
\hline Síndrome de Alport & 1 & 0,3 \\
\hline Síndrome de Pfeiffer tipo 2 & 1 & 0,3 \\
\hline Síndrome de Cohen & 1 & 0,3 \\
\hline Síndrome de Treacher Collins & 1 & 0,3 \\
\hline Síndrome de Noonan & 1 & 0,3 \\
\hline Síndrome de displasia frontonasal & 1 & 0,3 \\
\hline Síndrome de microdeleção 1 p32.3 e microduplicação 15 p11.2 & 1 & 0,3 \\
\hline Síndrome de microdeleção $2 \mathrm{q} 36.1 \mathrm{q} 36.3$ & 1 & 0,3 \\
\hline Genodermatose/hamartomatose & 35 & 12,1 \\
\hline Neurofibromatose tipo 1 & 25 & 8,6 \\
\hline Incontinência pigmentar & 3 & 1,0 \\
\hline Displasia ectodérmica não especificada & 2 & 0,7 \\
\hline Síndrome de Klippel Trenaunay Weber & 2 & 0,7 \\
\hline Epidermólise bolhosa autossômica recessiva & 1 & 0,3 \\
\hline Síndrome de Gorlin & 1 & 0,3 \\
\hline Hipomelanose de Ito & 1 & 0,3 \\
\hline Doenças predominantemente neurogenéticas & 23 & 7,9 \\
\hline Distrofia muscula de Duchenne & 8 & 2,7 \\
\hline Neuropatia sensitivo motora hereditária autossômica dominante não especificada & 3 & 1,0 \\
\hline Miopatia congênita não especificada & 4 & 1,4 \\
\hline Ataxia espinocerebelar familiar autossômica dominante não especificada & 2 & 0,7 \\
\hline Distrofia miotônica de Steinert & 2 & 0,7 \\
\hline Amiotrofia espinhal progressiva tipo 1 & 1 & 0,3 \\
\hline Ataxia de Friedreich & 1 & 0,3 \\
\hline Doença de Huntington & 1 & 0,3 \\
\hline Leucodistrofia metacromática & 1 & 0,3 \\
\hline Doenças predominantemente de tecido conjuntivo & 15 & 5,2 \\
\hline Síndrome de Marfan & 12 & 4,1 \\
\hline Síndrome de Ehlers-Danlos & 3 & 1,0 \\
\hline Síndromes de câncer hereditário & 11 & 3,8 \\
\hline Câncer de mama e ovário hereditários & 6 & 2,1 \\
\hline Polipomatose adenomatosa intestinal & 3 & 1,0 \\
\hline
\end{tabular}




\begin{tabular}{|c|c|c|}
\hline DIAGNÓSTICO GENÉTICO & $\mathbf{N}$ & $\%$ \\
\hline Retinoblastoma familiar & 1 & 0,3 \\
\hline Síndrome de Li Fraumeni & 1 & 0,3 \\
\hline Osteocondrodisplasias & 9 & 3,1 \\
\hline Acondroplasia & 2 & 0,6 \\
\hline Osteogênese imperfeita & 2 & 0,6 \\
\hline Picnodisostose & 1 & 0,3 \\
\hline Raquitismo hipofosfatêmico ligado ao X & 1 & 0,3 \\
\hline Displasia espondiloepifisária ligada ao X & 1 & 0,3 \\
\hline Displasia espondilometaepifisária congênita não especificada & 1 & 0,3 \\
\hline Nanismo tanatofórico & 1 & 0,3 \\
\hline Defeitos sensoriais & 8 & 2,7 \\
\hline Síndrome de Usher & 3 & 1,0 \\
\hline Surdez com herança autossômica recessiva não especificada & 2 & 0,6 \\
\hline Surdez com herança autossômica dominante não especificada & 1 & 0,3 \\
\hline Retinose pigmentar com herança autossômica recessiva & 1 & 0,3 \\
\hline Neuropatia óptica hereditária de Leber & 1 & 0,3 \\
\hline Defeitos de diferenciação e determinação sexual & 3 & 1,0 \\
\hline Hermafroditismo verdadeiro 46,XX & 1 & 0,3 \\
\hline Síndrome de Morris & 1 & 0,3 \\
\hline Síndrome de Rokitansky & 1 & 0,3 \\
\hline Sequências ou associações & 3 & 1,0 \\
\hline Displasia septo-ótica & 1 & 0,3 \\
\hline Associação VACTERL & 1 & 0,3 \\
\hline Sequência de Pierre Robin & 1 & 0,3 \\
\hline
\end{tabular}

Tabela 4: Distribuição dos pacientes com malformações congênitas isoladas, atendidos no Ambulatório de Genética Médica de São Carlos, SP, entre 2006 e 2018, nos quais foi definido um diagnóstico genético (N=48).

\begin{tabular}{l|r|r}
\hline \multicolumn{1}{c|}{ DIAGNÓSTICO GENÉTICO } & N & \multicolumn{2}{c}{$\%$} \\
\hline Malformações congênitas isoladas & 16 & 5,5 \\
\hline Defeito de sistema nervoso central & 9 & 3,1 \\
\hline Defeito da face & 9 & 3,1 \\
\hline Defeito cardíaco & 9 & 3,1 \\
\hline Defeito de membro & 3 & 1,0 \\
\hline Defeito de tórax, abdômen ou aparelho digestório & 2 & 0,6 \\
\hline Defeito genitourinário & & \\
\hline
\end{tabular}

A Figura 3 apresenta a resolubilidade do Ambulatório. Após avaliação clínica, a presença de doença genética foi afastada em 153 casos $(153 / 792$; 19,3\%). A maioria destes pacientes enquadrava-se em situação de abortamento de repetição $(43 / 153 ; 28,1 \%)$; casos de crianças com baixa estatura familiar e/ou constitucional, ou ainda, baixa estatura patológica de causa não genética

Figura 3: Resolubilidade do Ambulatório de Genética Médica de São Carlos, SP, entre 2006 e 2018.
$(37 / 153 ; 24,2 \%)$; crianças com dismorfias faciais, sem disfunções ou malformações maiores, consideradas eutróficas $(25 / 153$; $16,3 \%$ ); situações de deficiência intelectual e/ou defeitos congênitos em função de teratogênese $(13 / 153 ; 8,5 \%)$; e pessoas com deficiência intelectual secundária a anóxia neonatal $(10 / 153 ; 6,5 \%)$. 


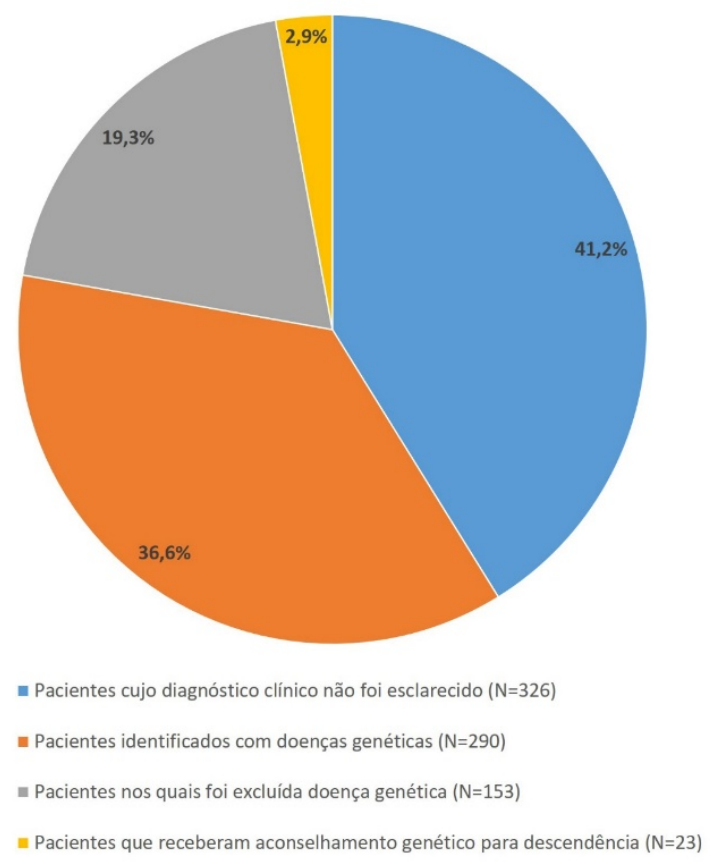

Em 326 pacientes $(41,2 \%)$ um diagnóstico genético não foi definido nem afastado. A maioria destes pacientes eram pessoas com deficiência intelectual isolada ou com pequenas dismorfias faciais $(174 / 326 ; \quad 53,4 \%)$; situações de malformações múltiplas, incluindo ou não deficiência intelectual, sem diagnóstico sindrômico estabelecido (77/326;23,6\%); crianças com atraso global de desenvolvimento isolado (37/326; 11,3\%); e crianças com transtorno de espectro do autismo sem deficiência intelectual associada (17/326; 5,2\%).

Vinte e três pacientes $(2,9 \%)$ receberam aconselhamento genético para futura descendência. Entre estes, nove pacientes tinham antecedente de óbito perinatal de feto com diagnóstico de doença genética, seis pacientes tinham história familiar positiva de doença genética previamente diagnosticada, seis pessoas procuraram o Ambulatório para aconselhamento genético pré-concepcional por causa de consanguinidade e duas pessoas em função de idade materna avançada.

\section{Discussão}

O Ambulatório de Genética de São Carlos iniciou suas atividades em junho de 2006. Na época, o Departamento de Medicina da UFSCar havia sido recémcriado e os professores mantinham relacionamento próximo com os gestores e frequentavam os equipamentos de saúde do município. $\mathrm{O}$ convite para abertura do Ambulatório, que funcionou desde o início como uma atividade de integração docenteassistencial, partiu da coordenadora do Centro Municipal de Especialidades em Saúde (CEME). Naquele momento, foram pactuadas as condições de trabalho, relacionadas a espaço físico, cronograma de atendimentos, número de vagas disponibilizadas, colaboração das equipes de enfermagem, de serviço social e de apoio administrativo do CEME, além do acesso ao cariótipo com bandeamento $\mathrm{G}$ para os pacientes com indicação clínica.

Considerando-se a população de abrangência e a prevalência das doenças genéticas, estima-se que cerca de 15.900 a 37.100 cidadãos, residentes nas cidades compreendidas pelo Ambulatório de 
Genética Médica de São Carlos, necessitarão de atenção à saúde na área da genética em algum momento das suas vidas. O Ambulatório de Genética Médica de São Carlos supriu parte das necessidades dessa população durante o período de seu funcionamento. Contudo, muitos pacientes não foram identificados como pessoas que se beneficiariam de atendimento na área da genética ou não tiveram acesso ao Ambulatório.

A suspeição de doenças genéticas ou de situações de risco para o seu desenvolvimento é uma competência esperada por parte dos profissionais de saúde que atuam na atenção básica, que nem sempre é suficientemente desenvolvida ${ }^{3,14,15}$. A utilização de instrumentos, como questionários e fichas, que facilitem o reconhecimento de fatores de risco e de situações suspeitas, de forma sistematizada, tem sido apontada como uma estratégia útil a ser incorporada pela rede de atenção básica para facilitar a identificação de pessoas com doenças genéticas, o que permite o encaminhamento regulado para serviços especializados ${ }^{16,17}$.

$\mathrm{O}$ acesso a atenção especializada em genética é um outro problema. O número de serviços de genética médica no país é insuficiente $^{6,7} \mathrm{e}$, apesar da maioria destes serviços estarem vinculados a instituições de ensino públicas, nem sempre estão inseridos nos sistemas de regulação regionais e estaduais da rede SUS. Situações nas quais os pacientes são encaminhados para os serviços de maneira informal, por meio de comunicação direta com os médicos geneticistas responsáveis pelos atendimentos nestes serviços, não são incomuns. Alternativas descritas na literatura para driblar a falta de especialistas em genética no país incluem a organização de serviços de genética itinerantes ${ }^{16}$ e o uso da telemedicina ${ }^{18}$.

Em relação ao fluxo de atendimentos do Ambulatório de Genética Médica de São Carlos ao longo dos anos, chama atenção a ausência de novos pacientes atendidos no ano de 2011. Isso aconteceu porque, neste ano, uma das professoras responsáveis pelos atendimentos esteve afastada. Na ocasião, houve tentativa de substituição desta médica por meio de concurso público municipal para médico geneticista ${ }^{19}$, mas não houve candidatos interessados na vaga. Genética é a especialidade médica com menor número de especialistas no Brasil ${ }^{4}$. Embora o Ministério da Saúde considere a especialidade prioritária, existem apenas 11 programas de Residência Médica em Genética no país, que oferecem, no total, 27 novas vagas para médicos residentes por ano e, à exceção de um programa vinculado à Secretaria de Estado de Saúde do Distrito Federal e outro programa vinculado à Universidade Federal da Bahia, todos os demais estão localizados nas regiões Sul e Sudeste $^{15}$. A formação de especialistas na área tem, portanto, acontecido de forma lenta e desigual.

Os motivos de encaminhamento de pacientes ao Ambulatório de Genética de São Carlos evidenciaram a grande diversidade de doenças genéticas, que acometem pessoas em faixas etárias muito díspares e atingem diferentes órgãos e sistemas. A frequência de casos não resolvidos, explicita as dificuldades inerentes ao diagnóstico clínico genético, além das barreiras para acesso aos testes genéticos moleculares. Nas situações mais comuns de indefinição diagnóstica, que estão relacionadas a deficiência intelectual, atraso global do desenvolvimento infantil e presença de defeitos congênitos múltiplos, os pacientes se beneficiariam com a investigação sistemática da síndrome do Xfrágil, com a análise cromossômica por testes de microarray e pelo sequenciamento completo do exoma, como recomendado na literatura ${ }^{20}$. Em 12 anos de funcionamento do Ambulatório, alguns pacientes tiveram acesso aos testes genéticos moleculares, por meio de projetos de pesquisa ${ }^{21-23}$, ou porque conseguiram negociar a realização dos testes diretamente junto à Secretaria 
Municipal de Saúde através de processos administrativos, ou ainda por meio de judicialização do SUS. No entanto, a oferta e o acesso aos testes genéticos moleculares aconteceram de forma irregular ao longo do tempo. Em particular, os pacientes que não residiam na cidade de São Carlos tiveram mais dificuldades para realizar os exames complementares, uma vez que os mesmos deveriam ser autorizados pelas Secretarias de Saúde dos seus municípios de origem.

Dificuldades diagnósticas adicionais foram percebidas nas situações de doenças neurogenéticas, nas quais muitas vezes o diagnóstico clínico foi estabelecido, mas o diagnóstico genético etiológico específico continuou inconclusivo, em grande medida por inacessibilidade a testes genéticos moleculares $^{24}$ e a biópsia muscular com estudo anatomopatológico adequado ${ }^{25}$. Existiram também cinco situações de óbito perinatal de fetos com múltiplos defeitos congênitos, ou seja, fetos polimalformados, nas quais não foi possível realizar necropsia, impossibilitando a definição diagnóstica sindrômica e prejudicando o aconselhamento genético familiar ${ }^{26}$.

Desde o lançamento da Política Nacional de Atenção Integral às Pessoas com Doenças Raras houve várias tratativas entre a coordenação do Ambulatório e a Secretaria Municipal de Saúde de São Carlos com intuito de viabilizar a habilitação do Ambulatório junto ao Ministério da Saúde, como um "Serviço de Atenção Especializada em Doenças Raras", em consonância com a Política Nacional de Atenção Integral às Pessoas com Doenças Raras ${ }^{9}$. As negociações foram prejudicadas pelas mudanças de gestão municipal e pela falta de um plano de governança estável, de longo prazo, para a área da saúde no município. A gestão municipal tem papel fundamental na assistência à saúde da população; é nesse nível da gestão pública que as demandas dos cidadãos por atenção à saúde são explicitadas e, é esse nível de gestão que, com apoio dos governos estadual e federal, deve garantir a oferta e o acesso aos serviços, de acordo com as necessidades de saúde requeridas. Esse é um desafio que a gestão municipal tem no campo das políticas públicas ${ }^{27}$.

Com todas as contingências, o Ambulatório de Genética Médica de São Carlos manteve seu funcionamento regular até junho de 2018. A partir de julho de 2018, a administração municipal de saúde dificultou o funcionamento do Ambulatório, com limitações em relação ao espaço físico onde os atendimentos aconteciam, no CEME, e com burocratização para a solicitação do cariótipo convencional, exame complementar que até então era realizado rotineiramente. É possível hipotetizar que esses obstáculos estiveram relacionados a presença dos estudantes de medicina no CEME, o que modificava a dinâmica do ambiente, e ao aumento de demanda por testes genéticos moleculares, que originava cada vez mais processos administrativos e judiciais. Frente a este cenário, e percebendo prejuízo no cuidado à saúde oferecido aos pacientes, a coordenação do Ambulatório optou por suspender $\mathrm{o}$ ingresso de novos pacientes a partir de agosto de 2018 e interromper os atendimentos em dezembro de 2018. A gestão municipal foi oficialmente comunicada sobre a suspensão dos atendimentos com três meses de antecedência, para que pudesse reestruturar a linha de cuidado dos pacientes em seguimento longitudinal.

Em 2019, foi realizado novo concurso municipal para médico geneticista no município ${ }^{28}$ e, desta vez, um médico especialista foi aprovado, embora não tenha sido convocado para assumir a vaga até o momento. Há expectativa de que com a convocação desse profissional, o Ambulatório seja reaberto e a parceria com a Universidade, quiçá, retomada.

Este não é o primeiro relato de extinção de um serviço de genética médica no Brasil. Entre 1969 e 1989, o serviço de 
genética médica da Universidade Federal da Bahia teve grande impacto na saúde pública local. Entretanto, mudanças administrativas importantes reduziram-no por muitos anos a um pequeno consultório particular de genética $^{29}$, até a retomada da sua estruturação no início dos anos 2000. Atualmente, o Hospital Universitário Prof. Edgar Santos, em Salvador, é um dos 16 "Serviços de Referência em Doenças Raras" habilitados pela Política Nacional de Atenção Integral às Pessoas com Doenças Raras (SBGM, 2020). Em 1996, houve o fechamento do setor de genética do Hospital Infantil Menino Jesus, que funcionou durante quatro anos na cidade de São Paulo. O encerramento deste serviço se deu no contexto das mudanças no sistema de saúde municipal de São Paulo, por ocasião do Plano de Atendimento à Saúde ${ }^{30}$. Em ambas as ocasiões não existiam políticas públicas relacionadas à atenção em genética no SUS, como existem atualmente. Ainda hoje, observa-se que a existência de políticas públicas na área não garante a organização de uma rede de atenção e de uma linha de cuidado em saúde para os pacientes com doenças genéticas no SUS. Pessoas com doenças genéticas raras continuam enfrentando longos itinerários terapêuticos até $\mathrm{o}$ diagnóstico; $\mathrm{o}$ desconhecimento dos médicos não geneticistas sobre as doenças raras; as dificuldades de acesso a especialistas, a exames diagnósticos e complementares; e as dificuldades de acesso a medicamentos e insumos alimentares de alto custo ${ }^{10}$. Adicionalmente, o diagnóstico tardio muitas vezes impede o paciente de receber tratamento adequado e oportuno, impactando na sua qualidade de vida ${ }^{2,10}$.

\section{Conclusões}

Existe um senso comum equivocado de que doenças genéticas são raras e de que o cuidado à saúde na área implicaria em alto custo. No entanto, as doenças genéticas em sua totalidade acometem um grande número de pessoas de diferentes faixas etárias. Embora a disponibilidade de recursos humanos e material para atendimento em genética seja muito desigual nas várias regiões do Brasil, o cuidado à saúde adequado é um direito desses pacientes e pode ser alcançado com a efetiva implantação da Política Nacional de Atenção Integral às Pessoas com Doenças Raras no SUS.

A regulação em saúde na área da genética no país é um tema que precisa ser melhor estudado e compreendido. Experiências como a do Ambulatório de Genética Médica de São Carlos, São Paulo, mostram os obstáculos de se prestar um serviço de saúde adequado à população, reforçando a necessidade de organizar a rede de atenção em genética no SUS, respeitando-se as atribuições da atenção básica e habilitando-se os serviços de atenção especializada, para reduzir as iniquidades em saúde entre as pessoas com doenças genéticas raras no país.

\section{Agradecimentos}

À Pró-Reitoria de Extensão da UFSCar, pelo apoio institucional. À Sra. Blaranis Pauletto, pelo apoio junto à Secretaria Municipal de Saúde de São Carlos, São Paulo. A todos os funcionários do Centro Municipal de Especialidades em Saúde de São Carlos, São Paulo. Ao Conselho Nacional de Desenvolvimento Científico e Tecnológico, pela concessão de bolsa de Iniciação Científica para a primeira autora, por meio do Programa Institucional de Bolsas de Iniciação Científica da UFSCar (CNPq/PIBIC - UFSCar).

\section{Financiamento}

Pró-Reitoria de Extensão da UFSCar e CNPq/PIBIC - UFSCar.

\section{Conflitos de interesse}

Não existem conflitos de interesse. 


\section{Referências}

1. Jorde LB, Carey JC, Bamshad MJ (org). Conceitos e história: o impacto clínico das doenças genéticas. In: Genética Médica. 5a ed. Rio de Janeiro: GEN Guanabara Koogan; 2017. p.1-5.

2. Nguengang Wakap S, Lambert DM, Olry A, Rodwell C, Gueydan C, Lanneau V, Murphy D, Le Cam Y, Rath A. Estimating cumulative point prevalence of rare diseases: analysis of the Orphanet database. Eur J Hum Genet. 2020; 28(2):165-173.

3. Melo DG, Germano CMR, Porciúncula CGG, Paiva IS, Neri JICF, Avó LRS, Demarzo MMP, Galera FM. Qualificação e provimento de médicos no contexto da Política Nacional de Atenção Integral às Pessoas com Doenças Raras no Sistema Único de Saúde (SUS). Interface (Botucatu). 2017; 21(suppl.1):1205-16.

4. Scheffer M (coord). Demografia Médica no Brasil 2020. São Paulo: Departamento de Medicina Preventiva da Faculdade de Medicina da USP; Conselho Federal de Medicina; 2020. 312 p. Available from: https://www.fm.usp.br/fmusp/conteudo/DemografiaMedica2020_9DEZ.pdf.

5. Passos-Bueno MR, Bertola D, Horovitz DD, de Faria Ferraz VE, Brito LA. Genetics and genomics in Brazil: a promising future. Mol Genet Genomic Med. 2014; 2(4):28091.

6. Novoa MC, Burnham TF. Desafios para a universalização da genética clínica: o caso brasileiro. Rev Panam Salud Publica. 2011; 29(1):61-8.

7. Horovitz DD, de Faria Ferraz VE, Dain S, Marques-de-Faria AP. Genetic services and testing in Brazil. J Community Genet. 2013; 4(3):355-75.

8. Brasil, Ministério da Saúde, Gabinete do Ministro. Portaria No 81, de 20 de janeiro de 2009. Institui, no âmbito do SUS, a Política Nacional de Atenção Integral em Genética Clínica. Diário Oficial da União da República Federativa do Brasil, Brasília, 21 de janeiro de 2009. Available from:

http://bvsms.saude.gov.br/bvs/saudelegis/gm/2009/prt0081_20_01_2009.html.

9. Brasil, Ministério da Saúde, Gabinete do Ministro. Portaria No 199, de 30 janeiro 2014. Institui a Política Nacional de Atenção Integral às Pessoas com Doenças Raras, aprova as Diretrizes para Atenção Integral às Pessoas com Doenças Raras no âmbito do Sistema Único de Saúde (SUS) e institui incentivos financeiros de custeio. Diário Oficial da União da República Federativa do Brasil, Brasília, 12 de fevereiro de 2014. Available from: http://bvsms.saude.gov.br/bvs/saudelegis/gm/2014/prt0199_30_01_2014.html.

10. Iriart JAB, Nucci MF, Muniz TP, Viana GB, Aureliano WA, Gibbon S. From the search for diagnosis to treatment uncertainties: challenges of care for rare genetic diseases in Brazil. Cien Saude Colet. 2019; 24(10):3637-50.

11. SBGM, Sociedade Brasileira de Genética Médica e Genômica. [Internet]. Available from: https://www.sbgm.org.br 
12. Melo DG, Lessa ACO, Teixeira-Filho JL, Nhoncanse GC, Drizlionoks E, Klein C, Barbosa CAA. Perfil clínico-epidemiológico da genética médica no Sistema Único de Saúde: análise do município de São Carlos, SP. Boletim Epidemiológico Paulista. 2010; 7(75):4-15.

13. Brasil, Instituto Brasileiro de Geografia e Estatística (IBGE). IBGE Cidades. [Internet]. Available from: http://www.cidades.ibge.gov.br/

14. Melo DG, Paula PK, Rodrigues SA, Avó LRS, Germano CMR, Demarzo MMP. Genetics in primary healthcare and the National Policy on Comprehensive Care for People with Rare Diseases in Brazil: opportunities and challenges for professional education. Journal of Community Genetics. 2015; 6(3):231-40.

15. Melo DG, Silva AA, Husny ASE, Ferraz VEF. Perfil de Competência em Genética para Médicos do Brasil: uma Proposta da Sociedade Brasileira de Genética Médica e Genômica. Revista Brasileira de Educação Médica. 2019; 43(Suppl. 1):440-50.

16. Vieira DKR, Horovitz DDG, Llerena Jr JC. Avaliação genética itinerante de crianças e adolescentes com deficiência vinculadas à Estratégia Saúde da Família. Revista Brasileira de Medicina de Família e Comunidade. 2012; 7(24):196-203.

17. Santos CS, Kishi RGB, Costa DLG, Silva DSD, Narciso TRF, Avó LRS, Germano CMR, Sandes KA, Acosta AX, Melo DG. Identificação de doenças genéticas na Atenção Primária à Saúde: experiência de um município de porte médio no Brasil. Rev Bras Med Fam Comunidade. 2020; 15(42):2347.

18. Tavares DF, Bueno LSM, Teixeira TRG, Cardoso-Jr LM, Varrone GR. A Utilização de Telemedicina para o Aconselhamento Genético em Oncologia. Rev. Bras. Cancerol. 2020; 66(1):20200129.

19. São Carlos. Diário Oficial do Município. Publicado em 07 de abril de 2011. Available from:

http://www.saocarlos.sp.gov.br/images/stories/diario_oficial_2011/DO_070411_certific ado.pdf

20. Srivastava S, Love-Nichols JA, Dies KA, Ledbetter DH, Martin CL, Chung WK, Firth HV, Frazier T, Hansen RL, Prock L, Brunner H, Hoang N, Scherer SW, Sahin M, Miller DT; NDD Exome Scoping Review Work Group. Meta-analysis and multidisciplinary consensus statement: exome sequencing is a first-tier clinical diagnostic test for individuals with neurodevelopmental disorders. Genet Med. 2019; 21(11):2413-2421.

21. Freitas ÉL, Gribble SM, Simioni M, Vieira TP, Prigmore E, Krepischi AC, Rosenberg C, Pearson PL, Melo DG, Gil-da-Silva-Lopes VL. A familial case with interstitial 2q36 deletion: variable phenotypic expression in full and mosaic state. Eur J Med Genet. 2012; 55(11):660-5.

22. Sabbag ARC, Rocha BG, Avó LRS, Germano CRM. Matheucci-Jr E, Melo DG. Identifying Microdeletion Syndromes in Patients with Intellectual Disability Using Molecular Genetic Testing: An Example for the Brazilian Public Health Care System. American Journal of Public Health Research. 2013; 1(4):86-92.

23. Araujo TF, Ribeiro EM, Arruda AP, Moreno CA, de Medeiros PF, Minillo RM, Melo DG, Kim CA, Doriqui MJ, Felix TM, Fock RA, Cavalcanti DP. Molecular analysis of 
the CTSK gene in a cohort of 33 Brazilian families with pycnodysostosis from a cluster in a Brazilian Northeast region. Eur J Med Res. 2016; 21(1):33.

24. Wofford S, Noblin S, Davis JM, Farach LS, Hashmi SS, Mancias P, Wagner VF. Genetic Testing Practices of Genetic Counselors, Geneticists, and Pediatric Neurologists with Regard to Childhood-Onset Neurogenetic Conditions. J Child Neurol. 2019; 34(4):177-183.

25. North KN, Wang CH, Clarke N, Jungbluth H, Vainzof M, Dowling JJ, Amburgey K, Quijano-Roy S, Beggs AH, Sewry C, Laing NG, Bönnemann CG; International Standard of Care Committee for Congenital Myopathies. Approach to the diagnosis of congenital myopathies. Neuromuscul Disord. 2014; 24(2):97-116.

26. Kültürsay N, Aşkar N, Terek D, Yeniel AÖ, Altun Köroğlu Ö, Yalaz M, Özkınay F, Akısü M. The Change of Perinatal Mortality Over Three Decades in a Reference Centre in the Aegean Region: Neonatal Mortality has decreased but Foetal Mortality Remains Unchanged. Balkan Med J. 2017; 34(6):553-558.

27. Cebes, Centro Brasileiro de Estudos de Saúde. A gestão municipal e a garantia do direito à saúde. Saúde debate. 2012; 36(95):506-11.

28. São Carlos. Diário Oficial do Município. Publicado em 10 de outubro de 2019. Available from: http://www.saocarlos.sp.gov.br/images/stories/diario_oficial_2019/DO_10102019_1454 .pdf

29. Souza MGF, Azevedo ES. Servicios de genética en las comunidades del Estado de Bahia, Brasil. Bol Oficina Sanit Panam 1993; 115(1):19-23.

30. Albano LM. Importância da genética no serviço público: relato da extinção de um setor de genética no Município de São Paulo, Brasil. Rev Panam Salud Publica. 2000; 7(1):2934.

\section{Como citar este artigo:}

Abreu T, Liberato ELA, Marsiglio HP, Santos TR, Ortensi NS, Barbosa CAA, Melo DG. Do surgimento à extinção: a trajetória de um serviço ambulatorial de genética médica no Brasil. Rev. Aten. Saúde. 2021; 19(68): 257-271. 